\title{
Non-Kolmogorov atmospheric turbulence and optical signal propagation
}

\author{
E. Golbraikh ${ }^{1}$, H. Branover ${ }^{1}$, N. S. Kopeika ${ }^{2}$, and A. Zilberman ${ }^{2}$ \\ ${ }^{1}$ Center for MHD Studies, P.O. Box 653, Ben-Gurion University of the Negev, Beer-Sheva 84105, Israel \\ ${ }^{2}$ Dept. of Electrooptical Engineering, P.O. Box 653, Ben-Gurion University of the Negev, Beer-Sheva 84105, Israel
}

Received: 26 January 2006 - Revised: 23 March 2006 - Accepted: 23 March 2006 - Published: 17 July 2006

\begin{abstract}
In the present review, we make an attempt to attract attention to the effect of non-Kolmogorov behavior of turbulence in various scales on the characteristics of electromagnetic waves propagation through a turbulent atmosphere on the example of certain atmospheric experiments. We discuss the interpretation of experimental data based on the model of spectral behavior of a passive scalar field within a broad range of scales, which has been developed recently.
\end{abstract}

\section{Introduction}

As known since long ago, atmospheric turbulence properties can considerably differ from those described by the classic Kolmogorov's theory, and in case of a passive scalar by Obukhov-Kolmogorov's (OK) theory. However, here we should specify that this observation refers, in the main, to the spectral behavior of structure functions of turbulent fields. Up to now, many works have been published examining nonKolmogorov's behavior of turbulent velocity field in various media and under various impacts. It becomes especially important at the study of the generation of large-scale coherent structures arising in the Earth's atmosphere. However, as a rule, spectral dependencies of structure functions of turbulent fluctuations on the wave vector (frequency) do not appear in these theories.

On the other hand, optics is one of significant areas where it is important to know these spectral dependencies, which are widely used. When studying the propagation of electromagnetic radiation through turbulent media, it becomes necessary to know spectral characteristics of both turbulent velocity and passive scalar fields (e.g., temperature or aerosol concentration fluctuations in the Earth's atmosphere) (Tatarski, 1967). Until now in practically 99\% of applied

Correspondence to: E. Golbraikh

(golbref@bgu.ac.il) studies, spectral characteristics of a turbulent passive scalar field are described by Obukhov-Kolmogorov's theory, which leads sometimes to a serious discrepancy between the theory and experimental data.

In the present review, we discuss some experimental data obtained both recently and rather long ago, on the propagation of electromagnetic waves in the Earth's atmosphere. We mention experimental results that have not been explained by classical OK theory and examine them on the basis of a broader approach comprising OK model modification and present-day studies of the spectral behavior of turbulent fluctuations at large scales.

\section{Some results of radio wave propagation in the Earth's atmosphere}

About 40 years ago (Tatarski, 1967), when discussing the results of the studies of long-range propagation of ultra-short radio waves in the Earth's troposphere, Bolgiano (1957) emphasized that the theory of electromagnetic waves scattering on atmospheric inhomogeneities satisfactorily explains various properties of a passing signal (rapid signal fading, drop of antenna power gain, etc.) (Tatarski, 1967). However, it was impossible to explain the dependence of the scattered field power on the frequency within the framework of this theory. The discrepancy between the theory and experimental data is due to the fact that while describing spectral characteristics of the turbulent field of atmospheric inhomogeneities using this theory, spectral density of the temperature field fluctuations obtained on the basis of ObukhovKolmogorov's theory was used. For the temperature turbulent field (which mainly affects the propagation of radio waves in the case under study), according to Obukhov (1949), the intensity of heat flux over the spectrum in the temperature field $\varepsilon_{T}$ and kinetic energy flux from large to small scales $\varepsilon_{u}$ are governing parameters in the dissipative

Published by Copernicus GmbH on behalf of the European Geosciences Union and the American Geophysical Union. 
and inertial intervals, and the correlation function of temperature $D_{T \varepsilon}(k)$ acquires the form:

$D_{T \varepsilon}(k)=c_{T \varepsilon}^{2} k^{-5 / 3}$,

where $c_{T \varepsilon}^{2}=$ const $\cdot \varepsilon_{T} / \varepsilon_{u}^{1 / 3}$.

Application of Eq. (1) for computing the dependence of the relative power of the scattered field $P(\lambda)$ on the wavelength $\lambda$ leads to

$P(\lambda) \sim \lambda^{-1 / 3}$.

However, special experiments (Bolgiano, 1957) have shown that in $99 \%$ of cases the exponent in Eq. (2) exceeds the value $-\frac{1}{3}$ (i.e., the exponent $-\gamma$ in Eq. (1) was below $-5 / 3$ ). Besides, in $50 \%$ of cases, the exponent in Eq. (2) exceeded 1 (i.e., $\gamma>3)$, and in $1 \%$ of cases - exceeded $2(\gamma>4)$. Its average value was close to $1(\gamma \approx 3)$. Thus, the theory referred to by Tatarski (1967) did not describe even qualitatively the behavior of $P(\lambda)$.

The existence of the spectrum of passive scalar fluctuations with $\gamma \approx 3$ in the Earth's troposphere was recently confirmed by experiments of lidar sounding of the troposphere (Zilberman et al., 2005) at the altitudes of $2-8 \mathrm{~km}$. It was obtained that spectra of similar type are observed in the troposphere starting from the altitudes around $6 \mathrm{~km}$. Besides, such spectra of atmospheric inhomogeneities fluctuations are characteristic of the stratosphere, too (Gurvich and Belen'kii, 1995; Belen'kii et al., 1997). The model suggested in these works combining temperature fluctuations at tropospheric altitudes in a "standard" OK way, and in the stratosphere - in a non-OK way, - is, apparently, the first modified OK model in the atmospheric optic studies.

Spectra with $\gamma=3$ in the turbulent velocity field have been known since long ago; they are characteristic of twodimensional turbulence. However, as to the passive scalar field, they are due to other factors. On the one hand, it is known (Gurvich and Belen'kii, 1995) that gravity waves lead to such fluctuation spectra. On the other hand, as demonstrated earlier by Elperin et al. (1996), the $\gamma=3$ spectrum is connected with the following fact: the behavior of the passive scalar field is determined only by parameters connected with the latter, whereas the velocity field parameters, e.g., such as the energy or helicity flux along the spectrum, do not affect it. Thus, it takes place only at $\operatorname{Pr}=v / D<1$ (where $v$ and $D$ are kinematic viscosity and molecular diffusion coefficient, respectively). In this case, this interval (with $\gamma=3$ ) appears within the scales $L_{B}<L<L_{C}$, where $L_{C}=\left(D^{3} / \varepsilon\right)^{1 / 4}$ is a scalar differential microscale, and $L_{B}=(D / \phi)^{1 / 2}$ at $\phi=(\varepsilon / \nu)^{1 / 2}$. However, this spectrum manifests itself most completely when the passive scalar distribution becomes anisotropic (although the turbulent velocity field remains homogeneous and isotropic) at $\operatorname{Pr}<1$. In this case, the interval $\gamma=3$ greatly expands up to $L_{u}>L>\max$ ( $\left.L_{C}, L_{K}\right)$, where $L_{u}$ is the characteristic scale of turbulent velocity field, and $L_{K} \sim \mathrm{Re}^{-3 / 4} L_{u}$ (where Re is the Reynolds number).
On the other hand, in the spectrum of the passive scalar we deal with different regions characterized by different governing parameters. If the parameter $\gamma$ acquires different values in different parts of the spectrum, we can speak about its effective value. Of course, in the experimental data we received the effective value of $\gamma$. As noted above, in about $50 \%$ of experimental cases $\gamma \geq 3$. Therefore, we can suppose that the spectral structure is more complicated than simply $\gamma=3$. Such spectrum can arise when, side by side with the inertial interval, even smaller scales $L<\max \left(L_{C}, L_{K}\right)$ affect the behavior of the scattered signal power. At $\operatorname{Pr}<<1$, an interval $L_{C}>L>L_{K}$ arises, where $\gamma \geq 17 / 3$ (Gibson and Schwartz, 1963; Gibson et al., 1988; Elperin et al., 1996; Golbraikh and Kopeika, 2004). And this steep slope in the small-scale part of the spectrum increases the effective slope of the structure function spectral density.

The influence of the small-scale interval with $\gamma \geq 17 / 3$ on the resulting effective spectrum (obtained experimentally), which can be written, according to (Elperin et al., 1996; Golbraikh and Kopeika, 2004) as

$D(k) \sim C_{1} \theta\left(k-k_{0}\right) k^{-3}+C_{2} \theta\left(k_{0}-k\right) k^{-17 / 3}$,

where $C_{1}$ and $C_{2}$ are constant depending on the passive scalar gradient, $k_{0} \sim \frac{1}{L_{C}}$, and $\theta\left(k-k_{0}\right)=\left(\begin{array}{c}1, k \geq k_{0} \\ 0, k<k_{0}\end{array}\right)$, depends on the value of $k_{0}$, which decreases with decreasing $\operatorname{Pr}$ (while the scale grows). Apparently, atmospheric conditions corresponding to a sufficiently strong warming at a weak wind were rarely observed in the experiments under consideration, where the effective value of the exponent $\gamma$ in Eq. (1) was practically close to 3 and very rarely reached 4 (approximately in $1 \%$ of cases).

Approximately at the same time, experiments on the study of phase fluctuations of centimeter radio waves were performed (Herbstreith and Thompson, 1955; Deam and Fannin, 1955; Norton, 1959). The route length was $18.5 \mathrm{~km}$; it passed at the altitudes of about 2000 to $4500 \mathrm{~m}$ above the sea level. The measured frequency spectrum of phase fluctuations of centimeter radio waves $W_{S}(f)$ was described to a good precision by the function $f^{-8 / 3}$ directly connected with the spectrum (1)

However, at the transition to spatial fluctuation spectra taking into account Taylor's hypothesis and mean wind velocity in experimental conditions $\approx 3 \mathrm{~m} / \mathrm{s}$, the authors came to the conclusion that this spectrum referred to large-scale fluctuations with the scales from $100 \mathrm{~m}$ to $10 \mathrm{~km}$, and significantly exceeded the external turbulence scale $L_{0} \approx 100 \mathrm{~m}$. Thus, in this case, spectrum (1) characteristic of the inertial interval describes also a large-scale spectrum of turbulent fluctuations of the passive scalar (Tatarski, 1967).

The behavior of large-scale turbulent fluctuations of the passive scalar near the outer scale $L_{0}$, as well as similar fluctuations of the velocity field, are until now an object of separate research that is far from being completed. 
On the other hand, as demonstrated by Branover et al. (2004), in the vicinity of the outer scale of a turbulent velocity field, a universal spectrum arises determined by an integral energy flux $\varepsilon_{V}=\int_{V} \varepsilon_{u} d V$. In this case, turbulent velocity field has an energy spectrum in the large scales $E(k) \sim k^{1 / 3}$. Assuming, as in case of the inertial interval, that in these scales $\varepsilon_{V}$ and $\varepsilon_{T}$ are determining parameters of the passive scalar field, we obtain the passive scalar spectrum in the vicinity of $L_{0}$ with $\gamma=8 / 3$ (Golbraikh and Kopeika, 2004). However, under atmospheric conditions, turbulent fluctuations of the velocity and passive scalar can be anisotropic. In the case, for example, of a quasi-twodimensional model, we can introduce two weakly connected integral fluxes: $\varepsilon_{V z}=\int_{z} \varepsilon_{u} d z$ in height and $\varepsilon_{V \perp}=\int_{S} \varepsilon_{u} d S$ over the area normal to $\mathrm{z}$ (Golbraikh and Kopeika, 2004).

At the same time, we can introduce governing parameters for passive scalar field in the outer scales: $\varepsilon_{T V}=\int_{V} \varepsilon_{T} d V$, and in an anisotropic case, $\varepsilon_{T Z}=\int_{z} \varepsilon_{T} d z$ and $\varepsilon_{T \perp}=\int_{S} \varepsilon_{T} d S$, by analogy with the velocity field (Golbraikh and Kopeika, 2004).

In a general case, the effect of the velocity field on the passive scalar field spaced over the scales. It means that, for instance, large-scale fluctuations of the velocity field can affect fluctuations of the passive scalar field from the inertial interval. Hence, outer scales of the velocity field and of the passive scalar can differ greatly, which leads to various combinations of the governing parameters (Golbraikh and Kopeika, 2004), and various types of spectra from a sufficiently steep one decreasing with $k(\gamma=8 / 3)$ at $L_{0 \text { Scalar }}<<L_{0 \text { Velocity }}$ to an increasing one $(\gamma=-4 / 3)$ at $L_{0 \text { Scalar }}>>L_{0 \text { Velocity }}$ can arise.

Thus, large-scale spectra of the atmospheric temperature fluctuations with $\gamma=5 / 3$, which were observed in the discussed experiments, arise when $\varepsilon_{V}$ and $\varepsilon_{T Z}$ become governing parameters in this region and are determined by the anisotropy of turbulent temperature field. This conclusion made on the basis of the study of governing parameters of turbulent fields in the troposphere completely agrees with the theory of electromagnetic waves scattering in the atmosphere. In fact (Tatarski, 1967), we know that at the scattering of radio waves, the wave vector of scattering equal to the difference of the wave vectors of the incident and scattered waves is, as a rule, directed vertically. This makes the influence of vertical structure of atmospheric inhomogeneities and their vertical spectra on this process rather essential. This is just what we have obtained in our analysis of the data on phase fluctuation spectra obtained in the above-described experiments.

\section{Optical experiments}

Obviously, if there exist deviations in the behavior of spectral characteristics of the passive scalar in the atmosphere, they should manifest themselves not only at the propagation of radio waves. We consider below some of these manifestations in optical experiments.

\subsection{Experiments using Shack-Hartmann wave-front sensor}

A Shack-Hartmann wave-front sensor constitutes a set of small lenses arranged on a common plane. Diameters of these lenses are much smaller than that of the telescope, which are A sensor located in the plane of the telescope pupil measures the average phase difference along each lens. Then, on the basis of this set of measurements, a phase pattern over the telescope pupil cross-section is made up taking into account all the lenses. This phase pattern makes it possible to evaluate the phase structure function (PSF). This method was used in various experiments aimed at the study of the effect of atmospheric turbulent fluctuations on the propagation of optical signals (Boreman and Dainty, 1996; Stribling et al., 1995; Nicholls et al., 1995; Rao et al., 1999).

As follows from these experiments, side by side with the OK behavior, NOK behavior of PSF is also observed. However, the fine structure of the structure function of the refractive index was not investigated in these works, but it was assumed that it has a certain index $\gamma$, which can, therefore, be regarded as the effective index $\gamma^{S H}$. On the other hand, we can gain some information about the fine structure, if we take into account that here the sub-aperture amounted, as a rule, to about $10-20 \mathrm{~cm}$. Hence, in these experiments, the structure function spectrum is limited from below by sufficiently small scales. As follows from experimental data, $\gamma^{S H}$ value is actually located within the above-mentioned interval of $5 / 3 \geq \gamma^{S H} \geq 1$.

Thus, the available experimental data indicate that under certain conditions, the effective spectrum of passive scalar fluctuations in the inertial interval becomes flatter than it follows from OK model. On the other hand, as demonstrated by Batchelor (1959) and Batchelor et al. (1959), at $\operatorname{Pr}>1$ in the vicinity of the diffusive interval, a region with $\gamma=1$ arises in the passive scalar spectrum. In this case, the "effective" $\gamma_{\text {eff }}$ magnitude of the entire spectrum, covering and $\gamma=1$ spectra becomes somewhat smoothed (Golbraikh and Kopeika, 2004).

Besides, in the range of relatively large scales that are usually considered in OK approximation, the passive scalar spectrum can be represented in the following form (Moiseev and Chkhetiani, 1996; Golbraikh and Moiseev, 2002) depending on helical properties of the velocity field turbulence:

$D_{T i}(k)=\frac{c_{T \varepsilon}^{2} k^{-5 / 3}}{1+\left(\frac{k}{k_{0}}\right)^{-1 / 3}}$.

where $k_{0}=\frac{\eta}{\varepsilon}, \eta=-\left(\frac{\partial H_{e}}{\partial t}\right)$ and $H=<\boldsymbol{u}^{\prime} \cdot \operatorname{rot} \boldsymbol{u}^{\prime}>$ is the mean helicity ( $\boldsymbol{u}^{\prime}$ is a turbulent component of the velocity field). In this case, "effective" exponent $\gamma_{\text {eff }}$ in the inertial interval 
varies from $5 / 3$ to $4 / 3$ and depends on the ratio $\frac{\eta}{\varepsilon}$, and the spectrum (3) is flatter than OK spectrum, which should lead to even greater flattening of $\gamma_{\text {eff }}$ (Golbraikh and Kopeika, 2004).

Thus, experimentally obtained values of the effective spectral index $5 / 3 \geq \gamma^{S H} \geq 1$ can be attributed to the fact that turbulent velocity field in the inertial interval, in contrast to purely Kolmogorov's case, is described by two parameters $\varepsilon_{u}$ and $\eta$, and not by $\varepsilon_{u}$ only. Besides, in the vicinity of the diffusive interval, an interval with $\gamma=1$ is formed. In combination with the inertial interval, this leads to the observed values of $\gamma^{S H}$.

\subsection{Studies of stellar jitter}

Another manifestation of NOK behavior of the atmospheric turbulence was found during the experiments on the motion of stellar image (stellar jitter) described by Belen'kii et al. (1997, 1999). Experimental data show that the dependence of tilt variance on the telescope diameter does not follow the predictions of the Kolmogorov model.

It is well known (Tatarski, 1967) that $\sigma^{2}$, mean square of relative fluctuations of luminous flux through the telescope, depends on its diameter $d$ and the structure function $D(\rho)$ as follows:

$\sigma^{2}(d)=\frac{1}{d^{2}} \int_{0}^{d}\left(\rho D^{\prime \prime}(\rho)+D^{\prime}(\rho)\right) f(\rho / d) d \rho$

where the apostrophe sign denotes derivatives with respect to $\rho$, and $f(\rho / d)$ is a certain function independent of $D(\rho)$. On the condition that spectral function describing turbulent atmospheric fluctuations obeys Eq. (1), $\sigma^{2}$ dependence on the diameter acquires a well-known form:

$\sigma^{2}(d)=A d^{-1 / 3}$

However, as demonstrated by Belen'kii et al. (1997, 1999), the experimental dependence $\sigma^{2}(d)$ is steeper than one obtained using Eq. (5), and at $d \geq 0.75 \mathrm{~m}$ it becomes slightly dependent on the aperture and even somewhat grows with increasing $d$. As the authors of these studies have pointed out, the application of Karman's model or semi-empirical model (Sasiela and Shelton, 1993) allows one to approximate the experimental data at $d \leq 0.7 \mathrm{~m}$ using the external scale parameter $L_{0} \approx 3-6 \mathrm{~m}$ for one model and $L_{0} \approx 6-12 \mathrm{~m}$ for another. However, no efforts allow the description of $\sigma^{2}(d)$ dependence at $d \geq 0.75 \mathrm{~m}$.

On the other hand, following Eq. (4) and introducing a certain effective value of the spectral index $\beta$ of the structure function $D(\rho)$ for $\sigma^{2}(d)$, we can write

$\sigma^{2}(d)=d^{\beta_{t f f}-2} \int_{0}^{d} F\left(x^{\beta_{t f f}-2}\right) d x$

where $F\left(x^{\beta_{\text {eff }}-2}\right)$ is a function of $\beta_{\text {eff }}$ and $x$ only. In Kolmogorov's case, $\beta_{\text {eff }}=5 / 3$, and we obtain Eq. (5).
It is noteworthy that with increasing telescope diameter $d$, the contribution of small-scale fluctuations decreases (Tatarski, 1967), and the role of large-scale ones grows. Therefore, von Karman's spectrum or semi-empirical specrum (Sasiela and Shelton, 1993) describe sufficiently well the behavior of $\sigma^{2}(d)$ at respective $L_{0}$. However, one should keep in mind that these spectra do not allow us to state that $L_{0}$ determined from experimental data constitutes an actual outer scale. But at the same time, relative anisotropy of $L_{0}$ is, apparently, actual.

Assuming that the spectral function in Eq. (8) can be described using $\beta=\beta_{\text {eff }}$ which can be represented in the form (Golbraikh and Kopeika, 2004):

$k^{-\beta_{e f f}} \sim \theta\left(k_{0}-k\right) k^{-\gamma_{1}}+B \theta\left(k-k_{0}\right) \theta\left(k_{D}-k\right) k^{-\gamma_{2}},(7)$

where $B$ is constant, $k_{0} \sim \frac{1}{L_{0}}, \gamma_{1}$ is the index $\gamma$ corresponding to the contribution of large scales, and $\gamma_{2}$ is the index $\gamma$ in the inertial interval. The wave vector value $k_{D}$ depends on the telescope aperture $\left(k_{D} \sim 1 / d\right)$ and, thus, determines the slope $\beta_{\text {eff. It follows from experimental data that in all cases }}$ the effective spectrum of a relatively small aperture is smaller than $\mathrm{OK}$ at $\beta_{\mathrm{eff}}=5 / 3$, and the slope of $\sigma^{2}(d)$ curves at $d<1-$ $1.5 \mathrm{~m}$ corresponds to the spectral index $5 / 3 \geq \beta_{\text {eff }}>4 / 3$, i.e. is close to Eq. (3).

On the other hand, assuming that in the saturation region (at $d \geq 1-1.5) \sigma^{2}(d)$ becomes a weak function of $d, \beta_{\text {eff }} \sim 2$ or is close to this value, which corresponds to the shift of the parameter $k_{0}$ towards smaller values or, respectively, larger scales. Integral power $\varepsilon_{V}$ (or its analogues $\varepsilon_{V z}$ and $\varepsilon_{V \perp}$ ) becomes one of governing parameters, which leads to $\sigma^{2}(d)$ flattening and even growth (Golbraikh and Kopeika, 2004).

\section{Conclusion}

In this brief review of the studies of the influence of atmospheric turbulence on electromagnetic waves propagation in the Earth's atmosphere, we have mentioned some wellknown experiments whose results remained unexplained within the framework of "standard" Obukhov-Kolmogorov's model. Of course, these are not the only experiments revealing the deviation of atmospheric turbulence from OK model. We have chosen them because these experiments cover practically the entire spectrum of scales of passive scalar fluctuations in the Earth's atmosphere. Depending on observation methods, problems to be solved, geophysical conditions, instruments applied and many other factors, different sections of the spectrum of passive scalar fluctuations (different sizes of inhomogeneities) affect electromagnetic radiation propagation through the atmosphere.

On the other hand, in many problems of radiation transfer through a turbulent medium, it is not necessary to know the fine structure of the passive scalar spectrum. A sort of its effective form is sufficient, since it appears in various integral convolutions in scales or frequencies. In our 
opinion, the development of such efficient semi-empirical models is among rather urgent problems. Here one should find a sufficiently universal spectral form that can take into account principal features of actual spectra in various scales.

Edited by: N. S. Erokhin

Reviewed by: two referees

\section{References}

Batchelor, G. K., Howells, I. D., and Townsend, A. A.: Small-scale variation of convected quantities like temperature in turbulent fluid. Part II. The case of large conductivity, J. Fluid Mech., 5, 134-139, 1959.

Batchelor, G. K.: Small-scale variation of convected quantities like temperature in turbulent fluid. Part I. General discussion and the case of small conductivity, J. Fluid Mech., 5, 113-133, 1959.

Belen'kii, M. S., Barchers, J. D., Karis, S. J., Osmon, C. L., Brown, J. M., and Fugate, R. Q.: Preliminary Experimental Evidence of Anisotropy of Turbulence and the Effect of Non-Kolmogorov Turbulence on Wavefront Tilt Statistics, Proc. SPIE, 3762, 396406, 1999.

Belen'kii, M. S., Karis, S. J., Brown, J. M., and Fugate, R. Q.: Experimental study of the effect of non-Kolmogorov stratospheric turbulence on star image motion, Proc. SPIE, 3126, 113-123, 1997.

Bolgiano Jr., R.: A meteorological interpretation of wavelength dependence in transhorison propagation, School of Electrical Engineering, Cornell Univ., Ithaca, New York, Res. Rept., EE 385, 1957.

Bolgiano Jr., R.: Spectrum of turbulent mixing, Phys. Rev., 108, 1348, 1957.

Boreman, D. and Dainty, C.: Zernike expansions for nonKolmogorov turbulence, J. Opt. Soc. Am. A, 13, 517-522, 1996.

Branover, H., Eidelman, A., Golbraikh, E., Gordienko, S., and Moiseev, S.: On the universality of large-scale turbulence, Phys. Fluids, 16, 845-847 2004.

Deam, A. P. and Fannin, B. M.: Phase-difference variation in 9350megcycle radio signals arriving at spaced antennas. Proc. IRE 43, N10, 1402, 1955.

Elperin, T., Kleeorin, N., and Rogachevskii, I.: Isotropic and anisotropic spectra of passive scalar fluctuations in turbulent fluid flow, Phys. Rev. E, 53, 3431-3441, 1996.
Gibson, C. H. and Schwarz, W. H.: The universal equilibrium spectra of turbulent velocity and scalar fields, J. Fluid Mech., 16, 365-384, 1963.

Gibson, C. H., Ashurst, W. T., and Kerstein, A. R.: Mixing of strongly diffusive passive scalars like temperature by turbulence, J. Fluid Mech., 194, 261-293, 1988.

Golbraikh, E. and Kopeika, N.: Behavior of structure function of refraction coefficients in different turbulent fields, Appl. Opt., 43, 6151-6156, 2004.

Golbraikh, E. and Moiseev, S. S.: Different spectra formation in the presence of helical transfer, Phys. Lett. A, 305, 173-175, 2002.

Gurvich, A. S. and Belen'kii, M. S.: Influence of stratospheric turbulence on infrared imaging, J. Opt. Soc. Am., A 12, 2517-2522, 1995.

Herbstreith, J. W. and Thompson, M. C.: Measurements of the phase radio waves received over transmission paths with electrical lengths varying as a result of atmospheric turbulence, Proc. IRE 43, N10, 1391, 1955.

Moiseev, S. S. and Chkhetiani, O. G.: Helical scaling in turbulence, JETP, 83, 192-198, 1996.

Nicholls, T. W., Boreman, G. D., and Dainty, C.: Use of a ShackHartmann wavefront sensor to measure deviations from a Kolmogorov phase spectrum, Opt. Lett. 20, 2460-2462, 1995.

Norton, K. A.: Recent experimental evidence favoring the $\rho \mathbf{K}_{1}(\rho)$ correlation function for describing the turbulence of refractivity in the troposphere and stratosphere, J. Atm. Terr. Phys., 15, 206, 1959.

Obukhov, A. M.: Temperature field structure in a turbulent flow, Izv. AN SSSR (Geogr. and Geophys. series), 13, 58-69 1949.

Rao, C., Jiang, W., and Ling, N.: Atmospheric parameters measurements for non-Kolmogorov turbulence with Shack-Hartmann wavefront sensor, Proc. SPIE, 3763, 84-91, 1999.

Sasiela, R. J. and Shelton, J. D.: Transverse spectral filtering and Mellin transform techniques applied to the effect of outer scale on tilt and tilt anisoplanatism, J. Opt. Soc. Am., A10, 646-660, 1993.

Stribling, B. E., Welsh, B. M., and Roggemann, M. C.: Optical propagation in non-Kolmogorov atmospheric turbulence, Proc. SPIE, 2471, 181-196, 1995.

Tatarski, V. I.: Wave Propagation in a Turbulent Medium, Nauka, Moscow, 1967.

Zilberman, A., Golbraikh, E., and Kopeika, N. S.: Lidar studies of aerosols and non-Kolmogorov turbulence in the Mediterranean troposphere, SPIE, 5987, 15-26. 2005. 